УДК 330.1

DOI: $10.14451 / 1.176 .7$

ГРНТИ 06.52.13

\title{
МАКРОЭКОНОМИЧЕСКАЯ СТАБИЛЬНОСТЬ И ДИНАМИКА ВАЛЮТНОГО КУРСА В МОДЕЛИ Р. ДОРНБУША
}

(c) 2019 Лихачев Михаил Олегович

доктор экономических наук, доцент, профессор кафедры Экономической теории и менеджмента Московский педагогический государственный университет, Россия, Москва

E-mail: olegmix71@mail.ru

В статье рассматривается модель открытой экономики, разработанная Р. Дронбушем с целью приоритетного управления колебаниями валютного курса и, связанными с ним колебаниями уровня цен. Автор рассматривает взаимосвязь базовых принципов модели Р. Дорнбуша с постулатами неоклассической макроэкономики и, в частности, монетаризма и оценивает ее применимость к современным экономическим условиям.

Ключевые слова: макроэкономическое равновесие, открытая экономика, монетаризм, валютный курс, модель Р. Дорнбуша, макроэкономическая политика, бюджетно-налоговая политика, денежнокредитная политика, макроэкономическая стабильность

Развитие современной мировой экономики характеризуется определенным исчерпанием идей и эффективных стратегий для дальнейшего развития. Мировой кризис 2008-2009 гг. и последовавшая за ним глобальная рецессия поставила под сомнения все существующие модели макроэкономического регулирования. Прежде всего это касается теорий и моделей неоклассического направления $[1 ; 4]$, господство которых, как считают многие современные экономисты, привело к тому, что системы государственного макроэкономического регулирования оказались не готовы к наступившему кризису. Однако использование кейнсианских методов [1; 4] борьбы с кризисом также не принесло значительных результатов. Мировая экономика так и не смогла вернуться к докризисным темпам роста и глобальная рецессия приняла затяжной характер. В этих условиях современной экономической науке необходимо подвергнуть ревизии весь имеющийся теоретический арсенал и в том числе неоклассические и монетаристские теории и модели, которые несмотря на свои существенные недостатки, несомненно, содержат рациональное зерно. В этом контексте модель валютного курса Р. Дорнбуша представляет несомненный интерес. Она по сути является полноценной моделью открытой экономики, увязывающей между собой колебания валютного курса, денежной массы, объемов национального производства и уровня цен.

\section{Модель Р. Дорнбуша}

Р. Дорнбуш разрабатывал свою модель для определения закономерностей, которым подчиняются краткосрочные колебания валютного курса $[2 ; 9 ; 10]$. Однако, поскольку валютный курс находится в определенной зависимости от основных макроэкономических величин, эта модель представляет собой, по сути, модель краткосрочного макроэкономического равновесия, которое устанавливается благодаря приближению текущих значений валютного курса к его долгосрочному, то есть равновесному значению. Эта модель разработана для экономической системы с плавающим валютным курсом и абсолютной мобильностью капитала и предполагает, что внутреннее равновесие достигается при стабильном уровне цен, то есть при нулевом темпе инфляции [8]. Выбор этих теоретических предпосылок, очевидно, обусловлен глобальными изменениями, происходящими в мировой валютной системе в связи с переходом к плавающим курсам валют и усилением влияния монетаризма на теорию и практику государственного регулирования.

Еще одна особенность модели Р. Дорнбуша заключается в том, что она в отличие от большинства макроэкономических моделей, основана на предположении о существовании нелинейных зависимостей между основными параметрами, характеризующими экономическую систему. Поэтому для исследования модели 
и формулирования условий ее устойчивости, все зависимости представлены в линеаризованной форме, то есть в виде логарифмических уравнений. Базовое соотношение модели описывает зависимость внутренней процентной ставки от ожидаемого обесценения национальной валюты в условиях абсолютной подвижности капитала:

$$
\mathrm{r}=\mathrm{r} *+\Theta(\overline{\mathrm{e}}-\mathrm{e})
$$

где $\mathrm{r}$ - ставка процента по активам номинированным в национальной валюте, $\mathrm{r}^{*}$ - среднемировое значение ставки процента на активы номинированные в иностранной валюте, $\overline{\mathrm{e}}-$ натуральный логарифм долгосрочного значения валютного курса, е - натуральный логарифм текущего валютного курса, а $\Theta$ - постоянный параметр, характеризующий влияние ожиданий относительно валютного курса на величину ставки процента. В основе этого лежит предположение о том, что активы «...номинированные в национальной и иностранной валюте являются совершенными субститутами друг друга, поэтому для уравновешивания ожидаемых изменений обменного курса устанавливается соответствующая премия. Таким образом, если ожидается обесценение национальной валюты, процентная ставка по активам, номинированным в национальной валюте, должна в той же пропорции превышать процентную ставку во внешнем мире» [2, с. 210].

Условия равновесия денежного рынка в модели Р. Дорнбуша задаются следующим логарифмическим уравнением:

$$
-\lambda r+\phi y=m-p
$$

где у - натуральный логарифм величины национального дохода, $\mathrm{m}$ - логарифм денежной массы, а $\mathrm{p}$ - логарифм уровня цен. С учетом того, что внутренняя ставка процента зависит от мировой ставки и от ожидаемого изменения курса, это выражение можно переписать как:

$$
\mathrm{p}-\mathrm{m}=-\phi \mathrm{y}+\lambda \mathrm{r} *+\lambda \Theta(\overline{\mathrm{e}}-\mathrm{e})
$$

В условиях равновесия, при стабильном объеме денежной массы, текущий курс валюты равен его долгосрочному значению и, следовательно, равновесный уровень цен может быть представлен как:

$$
\bar{p}=\mathrm{m}+(\lambda \mathrm{r} *-\phi \mathrm{y}) ;
$$

Вычитая из величины текущего уровня цен, значение его равновесного долгосрочного уровня, можно вывести уравнение, определяющее значение текущего валютного курса через величину долгосрочного курса и соотношение текущего и долгосрочного уровня цен:

$$
\mathrm{e}=\bar{e}-\frac{1}{\lambda}(\mathrm{p}-\bar{p})
$$

Разница между текущим и долгосрочным уровнем цен определяет разрыв между внутренней и мировой процентной ставкой. Если текущий уровень цен превышает долгосрочный равновесный уровень, это ведет к увеличению внутренней процентной ставки выше мирового уровня и вызывает приток капитала, который продолжается до тех пор, пока рост текущего валютного курса не приведет к тому, что ожидаемое обесценение национальной валюты в точности не уравновесится повышением внутренней процентной ставки [2, с. 212]. Поэтому при заданной величине долгосрочного валютного курса текущий уровень валютного курса определяется соотношением между текущим и долгосрочным равновесным уровнем цен.

Долгосрочные уровни валютного курса и уровня цен в модели Р. Дорнбуша определяются в соответствии с условиями равновесия на товарном рынке. Функция совокупного спроса имеет вид:

$$
\ln D=u+\delta(e-p)+\gamma y-\sigma r ;
$$

Это выражение отражает те же зависимости, что и кейнсианские модели совокупного спроса, основанные на тождестве национальных счетов. Так же, как и в тех моделях, совокупный спрос, здесь, находится в положительной зависимости от национального дохода и относительных цен отечественных товаров (e - p), что отражает эффект импортных закупок и в отрицательной зависимости от уровня внутренней процентной ставки.

Свою модель Р. Дорнбуш разработал для 
исследования краткосрочного эффекта воздействия денежно-кредитной политики на величину валютного курса. Поэтому основное внимание он сосредотачивает на исследовании неравновесных колебаний, которые происходят непосредственно после изменения объема денежной массы и до того, как товарный рынок успевает отреагировать на эти изменения. Поэтому он предполагает, что объем национального производства является фиксированной величиной и темп инфляции определяется степенью отклонения совокупного спроса от объема производства товаров и услуг в национальной экономике. Это сводит его теоретическую конструкцию к анализу краткосрочных неравновесных состояний. Однако, чтобы ограничить временные рамки рассматриваемых феноменов, Р. Дорнбуш должен был сформировать модель с более широкими временными горизонтами, которая включает не только реакцию денежного и валютного рынка на изменение денежной массы, но и реакцию объема национального производства на эти изменения. Эта расширенная версия модели Р. Дорнбуша представляет собой модель краткосрочного макроэкономического равновесия в открытой экономике.

В расширенной версии своей модели Р. Дорнбуш предполагает, что объем национального производства, определяется величиной совокупного спроса $(\mathrm{y}=\ln \mathrm{D})$. В этом случае уравнение, определяющее величину национального производства и дохода, будет выглядеть следующим образом:

$$
\mathrm{y}=\mu(\mathrm{u}+\delta(\mathrm{e}-\mathrm{p})-\sigma \mathrm{r}), \mu=1 /(1-\gamma)
$$

Темп инфляции определяется следующим выражением:

$$
\mathrm{p}^{\prime}=\pi(\mathrm{y}-\overline{\mathrm{y}})
$$

где $\mathrm{p}^{`}$ - темп инфляции, $\overline{\mathrm{y}}$ - долгосрочный объем национального производства («потенциальный выпуск»), который может интерпретироваться в кейнсианской версии как объем национального производства при полной занятости, либо в монетаристской версии как объем национального производства совместимый с естественным уровнем безработицы. В любом случае, превышение объема национального про- изводства над его долгосрочным равновесным значением вызывает инфляцию, а снижение текущего объема национального производства ниже его долгосрочного равновесного значения - дефляционное давление на уровень цен.

В условиях равновесия текущий объем национального производства равен «потенциальному выпуску», а внутренняя ставка процента равна мировой. Поэтому равновесную величину национального производства можно представить как:

$$
\overline{\mathrm{y}}=\mu(\mathrm{u}+\delta(\overline{\mathrm{e}}-\overline{\mathrm{p}})-\sigma \mathrm{r})
$$

Вычитая из текущего значения национального дохода его долгосрочное равновесное значение, и учитывая, что $\mathrm{r}^{*}-\mathrm{r}=\Theta(\mathrm{e}-\overline{\mathrm{e}})$, можно получить следующее выражение:

$$
y-\bar{y}=\mu(\delta+\sigma \Theta)(e-\bar{e})+\mu \delta(\bar{p}-p)
$$

Это уравнение представляет собой условие равновесия товарного рынка, выраженное через отклонение адаптационных параметров системы от равновесия, то есть через отклонение от равновесных значений объема национального производства, валютного курса и уровня цен.

Аналогично, вычитая из текущего уровня цен их долгосрочный равновесный уровень можно выразить условия равновесия для денежного рынка через отклонения адаптационных параметров от равновесных:

$$
\varphi(y-\bar{y})+(\bar{p}-p)=\lambda \Theta(e-\bar{e})
$$

Эти уравнения составляют совместную систему, решив которую можно представить объем национального производства и валютный курс как функции уровня цен:

$$
\begin{aligned}
& y-\bar{y}=-w(p-\bar{p}), w=\frac{\mu(\delta+\sigma \Theta)+\lambda \Theta \mu \delta}{\varphi \mu(\delta+\sigma \Theta) \varphi+\lambda \Theta} \\
& e-\bar{e}=-\frac{1-\lambda \mu \delta}{\varphi \mu(\delta+\sigma \Theta) \varphi+\lambda \Theta}(p-\bar{p})
\end{aligned}
$$

В результате все параметры системы определяются масштабом отклонения текущего уровня цен от их долгосрочного равновесного уровня, который, при прочих равных условиях, опреде- 
ляется объемом денежной массы. Это относится не только к объему национального производства, но и к темпу инфляции, зависимость которого от разрыва текущего и долгосрочного уровня цен может быть выражена как:

$$
p^{`}=-\pi w(p-\bar{p})
$$

Если текущий уровень цен превышает долгосрочный, то темп инфляции будет отрицательным, то есть цены будут снижаться до тех пор, пока не достигнут равновесного уровня. А если текущий уровень цен ниже равновесного, то темп инфляции будет положительным, и текущий уровень цен будет увеличиваться, пока не достигнет равновесного уровня. А вместе с уровнем цен достигают своих равновесных долгосрочных значений объем национального производства и валютный курс. Следовательно, именно инфляция является тем механизмом, который приводит систему к равновесию, когда государство, используя инструменты денежнокредитной политики, изменяет объем денежной массы.

\section{Заключение}

Очевидно, что модель Р. Дорнбуша носит ярко выраженный монетаристкий характер [3; 5; 6; 7]. Анализ этой модели приводит к выводу, что равновесная величина объема национального производства не зависит от параметров го- сударственной политики и определяется производственными возможностями экономической системы. Изменяя количество денег, государство может вызвать кратковременные отклонения объема национального производства от его равновесного уровня. Однако это связано с отклонением от равновесного уровня цен, который является определяющим параметром экономической системы. Если параметры экономической системы соответствуют условиям устойчивого равновесия, то превышение текущего объема национального производства над равновесным уровнем будет прождать снижение уровня цен ниже равновесного значения и, наоборот, снижение объема национального производства ниже равновесного уровня вызывает рост уровня цен. В любом случае, инфляция или дефляция вызывает сближение текущих и равновесных значений уровня цен, объема национального производства и валютного курса и приводит экономическую систему в состояние равновесия. При таких свойствах модели изменение денежной массы в долгосрочном плане будет вызывать только изменение уровня цен и валютного курса, но не будет отражаться на величине национального производства и дохода. Этот вывод соответствует монетаристской концепции, обосновывающей неэффективность краткосрочной денежно-кредитной политики и утверждающей необходимость ее согласования с долгосрочными изменениями реального производства.

\section{Библиографический список}

1. Блауг М. Экономическая мысль в ретроспективе. М.: Дело ЛТД.,1994.

2. Дорнбуш Р. Ожидания и динамика обменного курса. / Этот изменчивый обменный курс. - М. 2001. с. 207229.

3. Кейган Ф. Чикагская школа // Экономическая теория / Под. ред. Дж. Итуэлла, М. Милгрейта, П. Ньюмена.M., 2004. с. 553-565.

4. Лейонхувуд А. Макроэкономическая теория в двадцатом столетии: основные вехи развития. //Вопросы экономики. 2006. № 11. с. 26-45.

5. Редер М. У. Чикагская школа // Экономическая теория / Под. ред. Дж. Итуэлла, М. Милгрейта, П. Ньюмена.M., 2004. с. 48-61.

6. Фридмен М. Оптимальное количество денег.// Количественная теория денег.- М. 1996. С. 40-102.

7. Фридмен М. Количественная теория денег: новая формулировка // Количественная теория денег.- М. 1996. C. 18-39. 
8. Akiba H. Expectations, stability, and exchange rate dynamics under the Post Keynesian hypothesis // Journal of Post Keynesian Economics. Volume 27, 2004. pp. 125-140

9. Dornbusch R. Exchange Rates and Inflation MIT Press, 1988.

10. Dornbusch R. Keys to Prosperity: Free Markets Sound Money and a Bit of Luck, MIT Press, 2000. 